\title{
Joint Channel Estimation and Decoding with Low-Complexity Iterative Structures in Time-Varying Fading Channels
}

\author{
Yavuz Yapıcı \\ Dept. of Electrical and Electronics Eng. \\ Middle East Technical University \\ Ankara,TURKEY
}

\author{
A. Özgür Yılmaz \\ Dept. of Electrical and Electronics Eng. \\ Middle East Technical University \\ Ankara,TURKEY
}

\begin{abstract}
A low-complexity iterative channel estimation (ICE) algorithm is proposed with the promise of improved error performance. The new algorithm operates the LMS filter both in the forward and the backward directions along a block. The feedback from the decoder to the estimator is in the form of soft decisions. The pilot symbol assisted modulation (PSAM) is used as the transmission technique. The effect of code choice on various ICE algorithms is also explored by considering the blockwise concatenated codes initially offered for block-fading channels. The performance of the new estimation algorithm with the proposed coding is shown to outperform the conventional estimation algorithms over a fast time-varying Rayleigh fading channel beside its low complexity structure.
\end{abstract}

\section{INTRODUCTION}

Channel estimation is a major problem in digital communication systems especially for turbo codes operating at low signal-to-noise (SNR) ratio where the performances of most estimation algorithms are degraded. The iterative channel estimation (ICE) algorithm using the well-known iterative processing principle is a powerful candidate among the other channel estimation algorithms. We study a new ICE employing LMS algorithm with a proper coding over a time-varying narrowband fading channel. In this work, the previous works which may consider different channel models, transmission techniques and coding schemes are first overviewed to clearly remark our contribution.

In [1], an iterative receiver is proposed for fast timevarying frequency selective channels without any channel code. The proposed receiver employs a maximum-likelihood (ML) channel estimator using a known training sequence and a maximum-likelihood sequence estimator (MLSE) to extract the transmitted symbols. As an extension, the work in [2] considers a channel code over the same channel which is now assumed to be quasi-static. An LMS filter with hard estimates of the code symbols is used after the initial estimation iteration. However, any comparison showing the influence of using LMS algorithm instead of optimal ML estimator on the error performance is not provided.

This work was supported in part by the Scientific and Technological Research Council of Turkey (TUBITAK) under grant 104E027, and by STM Defence Technologies Engineering Inc.
The soft-decision feedback in ICE for a time-varying frequency selective channel is considered in [3]. The soft decisions of the code symbols together with the known training sequence are used to obtain an estimate of the channel by means of an LMS filtering. Similarly, the resulting error performance with LMS algorithm is not assessed by comparing it with that of minimum mean squared error (MMSE) estimation. The ICE with both hard and soft-decision feedback together with a MMSE type prediction filtering is discussed in [4]. In that work, the derived filter is not optimal with the usage of soft feedback. For slower fade rates, moving average filtering is suggested instead of MMSE to reduce the complexity. However, no simplification is proposed for fast time-varying channels.

The contribution of this paper is twofold. First, a lowcomplexity ICE is proposed which employs the LMS algorithm to estimate a fast time-varying narrowband fading channel. The LMS filtering is performed both in the forward and the backward directions to improve the performance by keeping the processing complexity close to the conventional LMS implementation. We prefer to use soft estimates of the code symbols with PSAM transmission technique. Second, the effect of code choice on different ICE algorithms is also explored. The blockwise concatenated codes proposed for blockfading channels and investigated over the ergodic channels by the present authors is offered as a powerful alternative [5]. The new low complexity ICE algorithm with the proposed coding is observed to outperform the recent conventional ICE algorithms, i.e., ICE with forward only LMS in [3] and ICE with MMSE approximation in [4]. It is also shown that the proposed blockwise coding achieve a better error performance than conventional codes with ICE algorithms.

The rest of the paper is organized as follows. In Section II, the channel model under consideration is introduced. The conventional ICE is reviewed and the new low-complexity estimation algorithm with improved error performance is proposed in Section III. The blockwise concatenated codes over time-varying fading channels is reviewed in Section IV. The computer simulation results are presented in Section V, and the paper ends up with some concluding remarks in Section VI. 


\section{System MODEL}

\section{A. Channel Model}

We consider the following discrete-time complex baseband equivalent time-varying fading channel model at time epoch $n$

$$
r_{n}=f_{n} a_{n}+v_{n}
$$

where $r_{n}$ is the received symbol, $f_{n}$ is a sample from a time-varying correlated fading process, $a_{n}$ is the transmitted symbol and $v_{n}$ is the sample from i.i.d. circularly symmetric Gaussian process with zero-mean and variance $\sigma_{v}^{2}$. We assume that the fading coefficients are unknown at the transmitter and the receiver, and are independent of the transmitted symbols and the noise. In addition, noise variance $\sigma_{v}^{2}$ and maximum Doppler frequency $f_{D}$ is assumed to be known a priori at the receiver.

In this work, we consider a frequency-flat Rayleigh fading process generated by using Jakes' isotropic scattering model [6]. In this model, the real and the imaginary parts of the complex channel coefficients are assumed to be independent with the following autocorrelation function

$$
r(k)=J_{0}\left(2 \pi f_{D} k T_{s}\right)
$$

where $J_{0}($.$) is the Bessel function of the first kind and$ zeroth order, and $T_{s}$ is the symbol duration. We also assume normalized flat fading, i.e., $E\left\{\left|f_{n}\right|^{2}\right\}=1$, and unit energy transmitted symbols, i.e., $E\left\{\left|a_{n}\right|^{2}\right\}=1$. The average received symbol SNR is

$$
\gamma_{s}=\frac{E\left\{\left|f_{n} a_{n}\right|^{2}\right\}}{E\left\{\left|v_{n}\right|^{2}\right\}}=\frac{E\left\{\left|f_{n}\right|^{2}\right\} E\left\{\left|a_{n}\right|^{2}\right\}}{\sigma_{v}^{2}}=\frac{1}{\sigma_{v}^{2}}
$$

and information bit SNR is $\gamma_{b}=\gamma_{s} / r$ where $r$ is the overall transmission rate of the system.

\section{B. Transmitter and Receiver Models}

Following the convention of [7], we use the pilot symbol assisted modulation (PSAM) in estimation of the flat-fading time-varying channel. In PSAM, known pilot symbols are inserted into the code stream prior to transmission as in Fig. 1. At the receiver, pilot symbols are used to obtain an estimate of the complex fading coefficients by employing a properly designed filter as shown in Fig. 2.

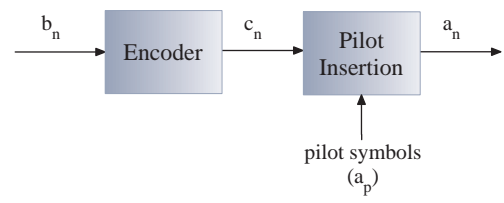

Fig. 1. Transmitter model

At the transmitter, a random data sequence $\left\{b_{n}\right\}$ is first encoded by an encoder as shown in Fig. 1. The resulting code stream $\left\{c_{n}\right\}$ is then split into frames of $(M-1)$ symbols and the known pilot symbols $\left\{a_{p}\right\}$ are inserted periodically into the center of each of the frame. We assume $a_{p}=1$ throughout the work without any loss of generalization. The parameter $M$ is called pilot symbol spacing and is assumed to be odd, as suggested in [7]. The code sequence $\left\{a_{n}\right\}$ including pilot symbols is then transmitted over a Rayleigh fading timevarying channel where all uncoded and coded sequences are in polar form, i.e., $\left\{a_{n}\right\},\left\{b_{n}\right\},\left\{c_{n}\right\} \in\{-1,1\}$.

At the receiver side, the ICE structure proposed in [4] is employed as shown in Fig. 2. As a first difference from the model considered in [4], the channel estimation algorithm derives an estimate for only the complex fading coefficients, not the noise variance $\sigma_{v}^{2}$ which is assumed to be provided to the receiver a priori. The received sequence $\left\{r_{n}\right\}$ is first processed using a properly designed filter to obtain an initial estimate of the complex fading channel coefficients. The loglikelihood ratios (LLRs) are then computed using the received sequence and the initial channel estimate. After removing the LLRs corresponding to the pilot symbols, the remaining LLRs are then passed to a soft-input soft-output (SISO) decoder.

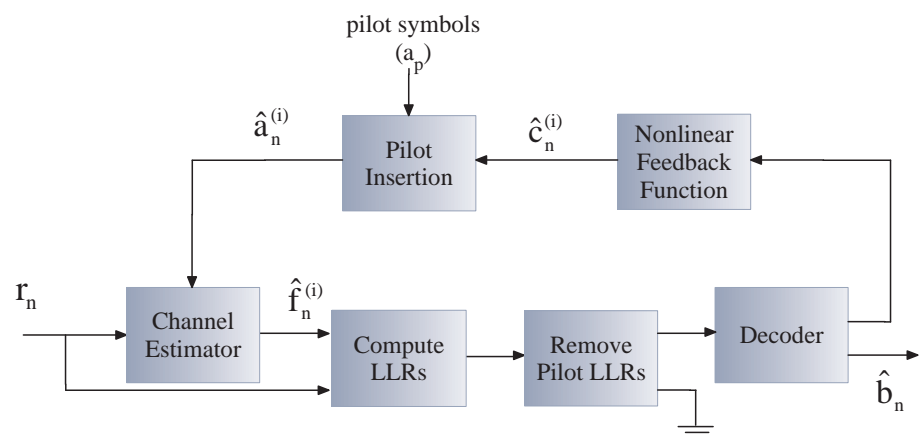

Fig. 2. Receiver model

The SISO decoder produces soft probabilities not only for the information symbols $\left\{b_{n}\right\}$ but also for the code symbols $\left\{c_{n}\right\}$. In the iterative channel estimation, these LLRs of the code symbols $\left\{\lambda_{n}^{(i)}\right\}$ are used to improve the estimation performance by treating the estimates of code symbols as if they were the known transmitted pilot symbols. For this purpose, $\left\{\lambda_{n}^{(i)}\right\}$ are passed through a nonlinearity to produce the soft-decision estimates of the code symbols where the superscript ${ }^{(i)}$ denotes the channel estimation iteration number. The pilot symbols are then inserted into the estimates of code symbols as in the transmitter, and the resulting stream is passed to the channel estimator. As a result, a new channel estimate is computed using not only the pilot symbols but also the estimated values of code symbols. As a second difference, we prefer to feed $\left\{\lambda_{n}^{(i)}\right\}$ back to the estimator not after each decoder iteration but after a sufficient number of decoder iterations so that the next channel estimate is computed using more reliable code symbol estimates. 


\section{Channel Estimation}

\section{A. Conventional Iterative Channel Estimation}

The observation model introduced in (1) may be modified for BPSK signalling to yield the following equivalent form

$$
\begin{aligned}
r_{n}^{\prime} & =a_{n} r_{n} \\
& =f_{n} a_{n}^{2}+a_{n} v_{n} \\
& =f_{n}+v_{n}^{\prime},
\end{aligned}
$$

where $v_{n}^{\prime}$ has exactly the same statistics as $v_{n}$, i.e., $E\left\{v_{n}^{\prime}\right\}=a_{n} E\left\{v_{n}\right\}=0$, and $E\left\{\left|v_{n}^{\prime}\right|^{2}\right\}=a_{n}^{2} E\left\{\left|v_{n}\right|^{2}\right\}=\sigma_{v}^{2}$ since $a_{n}^{2}=1$ in BPSK signalling.

In the data-aided mode where the transmitted symbols are known a-priori, the Wiener estimate of the fading coefficients may be computed by using a $(2 K+1)$-tap filter as

$$
\begin{aligned}
\hat{f}_{n} & =\sum_{k=-K}^{K} w_{k} r_{n-k}^{\prime}=\mathbf{w}^{T} \mathbf{r}_{n}^{\prime} \\
& =\sum_{k=-K}^{K} w_{k} a_{n-k} r_{n-k}
\end{aligned}
$$

where $\mathbf{w}=\left[w_{-K} w_{-K+1} \ldots w_{K}\right]^{T}$ is the tap-weight vector, and $\mathbf{r}_{n}^{\prime}=\left[\begin{array}{llll}r_{n+K}^{\prime} & r_{n+K-1}^{\prime} & \ldots & r_{n-K}^{\prime}\end{array}\right]^{T}$ is the modified observation vector both of which are of size $(2 K+1) \times 1$. We formulate the minimum mean square-error (MMSE) solution for the tap-weight vector $\mathbf{w}$ as follows

$$
E\left\{\mathbf{r}_{n}^{\prime H} \mathbf{r}_{n}^{\prime H}\right\} \mathbf{w}=E\left\{\mathbf{r}_{n}^{\prime} f_{n}^{*}\right\}^{1},
$$

which is indeed known as the Wiener-Hopf's system of linear equations. Using (4) in (7) yields the following matrix form

$$
\mathbf{w}=\left(\mathbf{R}+\sigma_{v}^{2} \mathbf{I}\right)^{-1} \mathbf{P} .
$$

where $\mathbf{R}$ is the $(2 K+1) \times(2 K+1)$ autocorrelation matrix of the fading coefficients with the entries $\mathbf{R}_{i j}=r(i-j)$, $\mathbf{P}=[r(K) r(K-1) \ldots r(-K)]^{T}$ is the $(2 K+1) \times 1$ crosscorrelation vector where the Jakes' fading autocorrelation function $r($.$) is defined in (2), and \mathbf{I}$ is the $(2 K+1) \times(2 K+1)$ identity matrix. Note that, the MMSE filter in (8) does not depend on the time index $n$, and therefore is exactly the same for all the fading coefficients in a transmitted block. This important facility is the reason behind using the modified observation model in (4).

In real communication scenario in which transmitted symbols are not known, the approximation

$$
\hat{f}_{n}=\sum_{k=-K}^{K} w_{k} a_{p} r_{p(n-k)}
$$

is suggested in [4] for the initial channel estimation where $r_{p(n-k)}$ is defined to be the observation closest to $r_{n-k}$, and

\footnotetext{
${ }^{1}()^{H}$ and ()$^{*}$ stand for Hermitian transpose and complex conjugate operations, respectively.
}

the index $p(n-k)$ is defined accordingly as

$$
p(n-k)=M\left\lfloor\frac{n-k}{M}\right\rfloor+\left(\frac{M+1}{2}\right) .
$$

After the initial channel estimation, the complex fading coefficients are suggested to be estimated using

$$
\hat{f}_{n}^{(i)}=\sum_{k=-K}^{K} w_{k} \hat{a}_{(n-k)}^{(i-1)} r_{n-k}
$$

for the $i$-th estimation iteration. Although the hard-decision feedback may be considered, this work focuses on the softdecision feedback operating according to

$$
c_{n}^{(i)}=\tanh \left(\lambda_{n}^{(i)}\right)
$$

which in fact corresponds to the expected value from the LLR. Note that, the filtering operation in (11) which is proposed in [4] is not optimal from MMSE point of view since $\left(\hat{a}_{(n-k)}^{(i)}\right)^{2} \neq 1$ in soft decision feedback mechanism. Nevertheless, this approach is employed in this work for its practicality and comparison purposes with the recent works.

\section{B. Low-Complexity Iterative Channel Estimation with LMS}

In this section, we propose a low-complexity ICE algorithm with improved error performance which employs the wellknown LMS algorithm [8].

The proposed algorithm derives an initial channel estimate according to (9) by using pilot symbols only. In the subsequent channel estimation iterations, an LMS filter is employed instead of MMSE filtering to reduce the overall processing complexity. The soft estimates of the code symbols are also incorporated into the channel estimator together with the pilot symbols, as before.

Two different approaches are considered while employing the LMS algorithm to perform channel estimation. The first approach, which we call l-way LMS, utilizes the LMS algorithm in a conventional manner where an estimate of the channel at the $i$-th estimation iteration is computed using

$$
\hat{f}_{n+1}^{(i)}=\hat{f}_{n}^{(i)}+\beta e_{n}^{(i)} \hat{a}_{n}^{(i-1)}
$$

where the complex conjugate operation on the estimate of the real transmitted symbol is omitted and $\beta$ stands for step size of the algorithm. Note that, in order to implement the LMS algorithm at the $i$-th channel estimation iteration, the estimate of the transmitted symbols at the $(i-1)$-st estimation iteration is used. The error term $e_{n}^{(i)}$ in (13) is defined as

$$
e_{n}^{(i)}=r_{n}-\hat{f}_{n}^{(i)} \hat{a}_{n}^{(i-1)} .
$$

The second approach, which we call 2-way LMS, operates the LMS algorithm both in the forward and the backward direction along the transmitted block. We define $\left\{h_{n}\right\}$ to be the estimate of complex fading channel coefficients produced by the LMS algorithm operating in the forward direction, and $\left\{g_{n}\right\}$ to be the estimate in the backward direction. The overall channel estimate used in LLR computations is the arithmetic 
average of the forward and backward estimates at each time epoch $n$.

The forward LMS algorithm is identical to 1-way LMS operating in the forward direction, and $\left\{h_{n}\right\}$ is therefore computed according to (13)-(14). Similarly, the backward LMS algorithm is equivalent to the 1-way LMS in the backward direction, and operates according to

$$
g_{n-1}^{(i)}=g_{n}^{(i)}+\beta e_{b, n}^{(i)} \hat{a}_{n}^{(i-1)}
$$

where the error term $e_{b, n}^{(i)}$ is defined as

$$
e_{b, n}^{(i)}=r_{n}-g_{n}^{(i)} \hat{a}_{n}^{(i-1)} .
$$

The resulting complex fading channel coefficients are computed by averaging the forward and backward estimates at each time epoch $n$ as follows

$$
\hat{f}_{n}^{(i)}=\frac{\left(h_{n}^{(i)}+g_{n}^{(i)}\right)}{2} .
$$

\section{Coding For Iterative Channel Estimation}

In [5], the present authors investigate the coding over both the block-fading and the time-varying narrowband fading channels where the former is a good model for the latter, in general. The blockwise concatenated convolutional code (BCCC) which is initially designed for the block-fading channels is shown to outperform the conventional serially concatenated convolutional code (SCCC) over the fully interleaved time-varying channels, as well.

In this work, we investigate the effect of code choice on the performance of ICE structures with various prediction filters. Indeed, since the ICE algorithms benefit from the decoded symbols to refine the channel estimate, the error performance of the codes being used is expected to have a major effect on the overall performance. We examine this subject by employing both the conventional SCCC as well as the powerful candidate BCCC in iterative channel estimation structures, and report the corresponding simulation results in the following section.

\section{Simulation Results}

The error performances of the aforementioned iterative estimation algorithms and channel codes are explored by performing extensive computer simulations. A fast time-varying Rayleigh flat-fading channel with a maximum normalized Doppler frequency of $f_{D} T_{s}=0.01$ is considered for this purpose. The transmitted information blocks are of 100 symbols long, and are encoded by either a SCCC or a BCCC code, both of which are composed of outer and inner convolutional encoders with the generator polynomials $(1,5 / 7)_{8}$ and $(1,1 / 3)_{8}$, respectively, in octal form. The trellis termination is also assumed by employing 2 bits and 1 bits for the outer and inner encoders, respectively.

The number of iterations for both channel estimation and decoder iterations is set to be 5 which provides a satisfactory convergence with $10^{5}$ independent trials at each SNR level. In

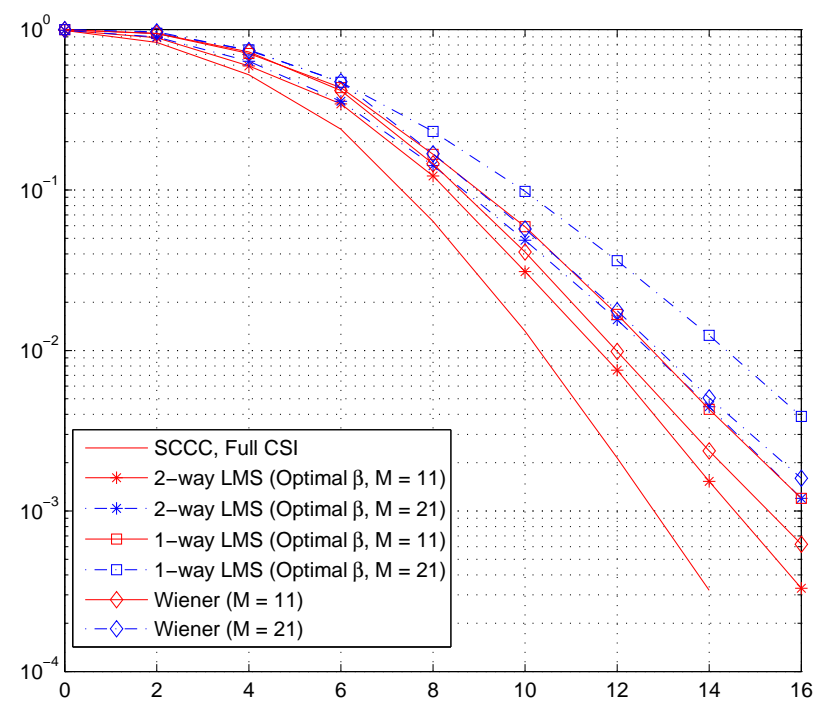

Fig. 3. BLER of SCCC over a fast Rayleigh flat-fading channel with the normalized fading rate $f_{D} T_{s}=0.01$, data sequence of length 100 , symbol spacing $M=11$ and $M=21$, and the filter size $2 K+1=21$. Both the 1-way LMS and 2-way LMS algorithms are operated with the best step-size $(\beta)$ values.

order to achieve the best performance, the subblock number $N$ of BCCC is set to 10 with the rationale explained in [5] in detail. In simulations, optimal step size $(\beta)$ values are used for both 1-way and 2-way LMS algorithms. The best $\beta$ is chosen from the set $\{0.1,0.15,0.2, \ldots, 0.5\}$ independently at each SNR value on trial and error basis. The initialization of LMS algorithm is performed by using the perfect channel estimates.

Throughout the simulations, we choose the MMSE filter size to be 21 , i.e., $K=10$, which is reasonable for our purpose which is to achieve a high performance ICE with low complexity. Therefore, although the error performance of MMSE is expected to improve with the increasing filter size, we avoid from such a scenario in order to compare the error performances on an acceptable level of complexity.

In the Fig. 3 and Fig. 4, we demonstrate the BLER performance of the estimation algorithms under consideration with different channel codes and pilot symbol spacing $(M)$ values. It is observed under all scenarios that the 2-way LMS is the best choice among the others. The 2-way LMS at BLER $=10^{-3}$ is off the known channel case by only $1 \mathrm{~dB}$ under BCCC encoding and $M=11$, whereas this gap is $1.8 \mathrm{~dB}$ for both the MMSE approximation and 1-way LMS under the same scenario. These SNR gaps are changed to be $1.75 \mathrm{~dB}, 2.45 \mathrm{~dB}$, and $3.60 \mathrm{~dB}$ for the 2-way LMS, the MMSE approximation and the 1-way LMS algorithms, respectively, when the SCCC channel code is employed instead, where the other conditions are kept unchanged. Note that, a 21-tap FIR filter with a high complexity is used for the MMSE prediction, whereas the computational complexity of 2-way 


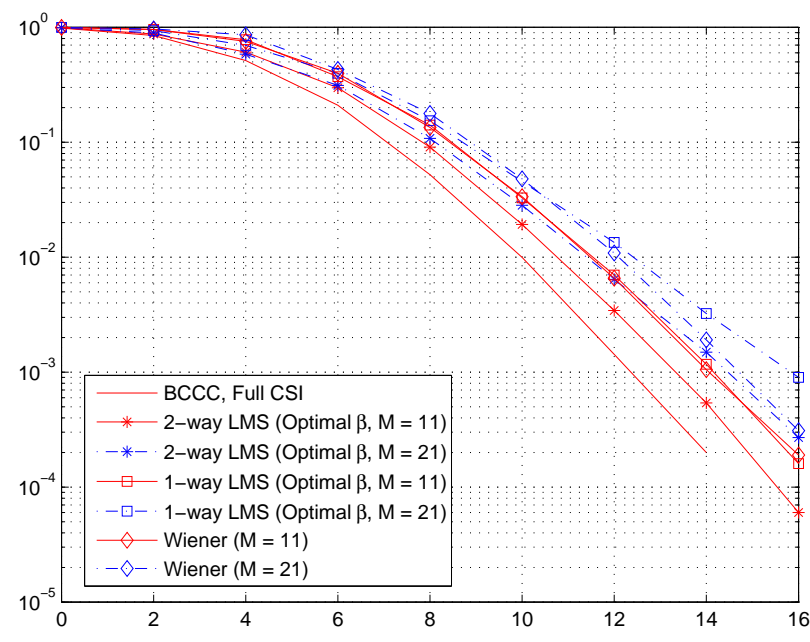

Fig. 4. BLER of BCCC over a fast Rayleigh fading channel with the normalized fading rate $f_{D} T_{s}=0.01$, data sequence of length 100 , symbol spacing $M=11$ and $M=21$, and the filter size $2 K+1=21$. Both the 1-way LMS and 2-way LMS algorithms are operated with the best step-size $(\beta)$ values.

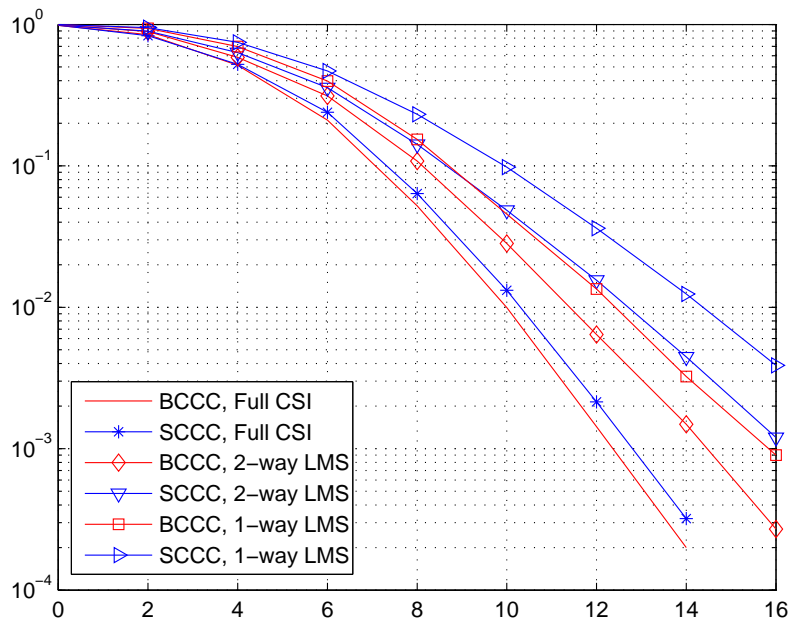

Fig. 5. BLER of BCCC over a fast Rayleigh fading channel with normalized fading rate $f_{D} T_{s}=0.01$, data sequence of length 100 , symbol spacing $M=21$ and the filter size $2 K+1=21$.

LMS is basically as low as the single-tap 1-way LMS filter.

Several comments may made on these results about the effect of the pilot density and the code choice. First of all, the error performance degrades with the decreased number of pilot symbols, i.e., when $M$ increases from $M=11$ to $M=21$, for both of the channel codes, as expected. Secondly, the robustness of the estimation algorithms under consideration are different with respect to the increase in $M$. For example, 2-way LMS requires extra $1.31 \mathrm{~dB}$ SNR to achieve BLER $=10^{-2.4}$ under SCCC encoding when $M$ increases from 11 to 21 , whereas MMSE approximation and 1-way LMS require $1.16 \mathrm{~dB}$ and $1.85 \mathrm{~dB}$, respectively, under the same scenario. The extra SNR requirement for 2-way LMS, MMSE approximation and 1-way LMS is observed to be $1.11 \mathrm{~dB}, 0.59 \mathrm{~dB}$, and $1.71 \mathrm{~dB}$, respectively, under the same scenario except for the BCCC encoding and BLER $=10^{-3}$.

In order to better evaluate the effect of the code choice, the BLER curves of 1-way and 2-way LMS estimation algorithms in the Fig. 3 and Fig. 4 are placed in the Fig. 5 for $M=11$. In this clear figure, it is observed that the performance gap between SCCC and BCCC is only $0.45 \mathrm{~dB}$ in the known channel case, whereas this value increases to $1.80 \mathrm{~dB}$ for 2-way LMS and $2.00 \mathrm{~dB}$ for 1-way LMS algorithms in the unknown channel case. Therefore, the error performance of the channel estimation algorithms are closer to the known channel performance when the BCCC code is employed instead of SCCC.

\section{SUMMARY}

A new low-complexity and improved performance iterative channel estimation algorithm is proposed for the fast Rayleigh flat-fading channels. The new algorithm employs the LMS filtering in both the forward and the backward directions to improve the error performance. Simulation results indicate a remarkable SNR improvement along with the reduced processing complexity as compared to the conventional approach with the MMSE based prediction. The error performance of the considered algorithms are also observed to depend on the code choice. The blockwise concatenated codes, which is initially designed for the block-fading channels, are explored over the fully correlated channel, and the corresponding error performances of the estimation algorithms are observed to be more closer to the known channel performance when it is compared with the case in which the conventional serially concatenated codes are employed.

\section{REFERENCES}

[1] K.-H. Chang and C. Georghiades, "Iterative joint sequence and channel estimation for fast time-varying intersymbol interference channels," in Proc. of IEEE ICC'95, vol. 1, Seattle, WA, Jun. 1995, pp. 357-361.

[2] N. Nefedov, M. Pukkila, R. Visoz, and A. Berthet, "Iterative data detection and channel estimation for advanced TDMA systems," IEEE Trans. Wireless Commun., vol. 51, no. 2, pp. 141-144, Feb. 2003.

[3] R. Otnes and M. Tuchler, "Iterative channel estimation for turbo equalization of time-varying frequency-selective channels," IEEE Trans. Wireless Commun., vol. 3, no. 6, pp. 1918-1923, Nov. 2004.

[4] M. C. Valenti and B. D. Woerner, "Iterative channel estimation and decoding of pilot symbol assisted turbo codes over flat-fading channels," IEEE J. Sel. Areas Commun., vol. 19, pp. 1697-1705, Sep. 2001.

[5] Y. Yapıcı and A. Ö. Yılmaz, "Turbo-like codes over block-fading and time-varying narrowband fading channels," in Proc. of IEEE VTC'09, Barcelona, Spain, Apr. 26-29, 2009.

[6] A. Goldsmith, Wireless Communications. New York, NY, USA: Cambridge University Press, 2005.

[7] J. K. Cavers, "An analysis of pilot symbol assisted modulation for rayleigh fading channels," IEEE Trans. Veh. Technol., vol. 40, pp. 686-693, Nov. 1991.

[8] J. Proakis, Digital Communications. McGraw-Hill, 2000. 